\title{
MODELOS DE CRISIS DE TIPO DE CAMBIO Y SU APLICACIÓN A LA CRISIS MEXICANA DE 1994
}

\author{
Humberto Banda Ortiz* \\ Tecnológico de Monterrey, Campus Santa Fe \\ (Recibido 3 de mayo 2004, aceptado 15 de junio 2004)
}

\section{Resumen}

En este trabajo se trata de determinar si los modelos teóricos sobre crisis cambiarias desarrollados hasta la actualidad son capaces de explicar la crisis financiera mexicana de 1994. Para ello se describen los tres principales conjuntos de modelos teóricos. Posteriormente se analiza cuáles fueron las características económicas más relevantes en los años anteriores a la crisis. Finalmente se analiza si lo descrito en el análisis empírico es compatible con lo explicado por los modelos teóricos.

\section{Abstract}

This essay tries to determine if the theoretical models about the exchange rate crisis developed up to today are capable of explaining the Mexican financial crisis of 1994 . In order to do this, we will begin by describing the three main theoretical models. Subsequently, it will analyze which were the most relevant economical characteristics in the years previous to the crisis. At the end we will analyze if the described in the empirical analysis is compatible with the theoretical models.

Clasificación JEL: E44, E65, F31

Palabras clave: Mercados financieros, Crisis cambiarias, Tipo de Cambio

* Departamento de Finanzas, Tecnológico de Monterrey, Campus Santa Fe. Av. Carlos Lazo No. 100. Col. Sta. Fe. Deleg. Cuajimalpa, C. P. 01389. México, D. F. Teléfono +52(55) 91778000 ext. 7781. Correo electrónico: humberto.banda@itesm.mx 


\section{Introducción}

A lo largo de las últimas tres décadas se han formalizado modelos teóricos que tratan de explicar las crisis de balanza de pagos. En la literatura estos modelos se clasifican en tres grupos: modelos de primera, de segunda y de tercera generación.

Los modelos de primera generación surgen con Krugman (1979) que adapta el modelo de Salant y Henderson (1978) sobre la fijación del precio de un bien no renovable al caso particular de la fijación del precio de una divisa. La hipótesis de estos modelos es que la crisis de balanza de pagos se produce por la incompatibilidad de la política cambiaría con las políticas fiscal y monetaria del gobierno.

Los modelos de segunda generación aparecen en 1994 con Obstfeld. Dichos modelos consideran que las crisis cambiarias surgen por las expectativas de los agentes privados sobre la gestión del gobierno que se autoconfirman.

Los modelos de tercera generación ponen de manifiesto la interrelación entre las crisis financieras y la debilidad de los sistemas financieros locales. Estos modelos tratan de caracterizar las crisis de los países del sudeste asiático. Los modelos de tercera generación no constituyen un campo unificado sino que ofrecen, más bien, explicaciones parciales de distintos elementos que caracterizaron las crisis asiáticas.

Los modelos de primera generación tienden a ajustarse a lo ocurrido en América Latina a principios de la década de los 80. Los modelos de segunda generación explican las crisis del Sistema Monetario Europeo de 1992 y 1993, así como la de México de 1994. Los modelos de tercera generación se ajustan a lo acaecido en Asia en 1997.

El objetivo de este artículo es determinar si los modelos teóricos a los que ha hecho referencia se ajustan a la evidencia empírica mexicana. Para ello, en primer lugar, se describen las premisas básicas de los modelos. En segundo lugar, se realiza un estudio empírico de la economía mexicana en los años previos a la crisis de 1994, incidiendo especialmente en aquellas variables que condujeron al colapso del régimen cambiario. Finalmente se observa si las explicaciones que se extraen del estudio empírico son compatibles con las explicaciones que los modelos ofrecen.

\section{Modelos teóricos que explican las crisis financieras}

\subsection{Modelos de primera generación}

Salant y Henderson (1978) propusieron un modelo en el que se fija el precio de un bien no renovable, por ejemplo el oro, y en el intento de que no se eleve dicho precio las autoridades venden oro, con lo que finalmente se agotarían sus reservas de dicho bien. En 1979 Krugman adapta el modelo de Salant y Henderson para explicar las crisis de balanza de pagos, que definen como el momento en el cual un gobierno tiene que abandonar su política cambiaría, consistente en mantener un ancla con respecto a una o más monedas extranjeras. A este modelo se adhirieron varios autores. Flood y Garber (1984) desarrollaron un modelo lineal con una variable estocástica, y posteriormente lo ampliaron con el concepto de la esterilización de la pérdida de reservas (Flood, Garber y 
Kramer (1996)). Connolly y Taylor (1984) analizaron un régimen de tipo de cambio crawling peg, en vez de un régimen de tipo de cambio fijo. Edwards (1989) hace hincapié en los patrones de apreciación de la moneda y deterioro de la balanza por cuenta corriente que suelen preceder a la devaluación de la moneda.

Los modelos de primera generación se basan en la incompatibilidad entre un sistema de tipo de cambio fijo y la política monetaria, como instrumento de la política de estabilización (con el objetivo de conseguir el equilibrio interno) y su función correctora de los desequilibrios de la balanza de pagos a través de la intervención en el mercado de cambios.

Los modelos de primera generación tienden a considerar que la creación excesiva de crédito interno tendría su origen en un déficit presupuestario excesivo, por lo que con anterioridad al ataque especulativo, se deberían observar unas políticas monetaria y fiscal expansivas. De esta manera, la creación de crédito interno por encima del crecimiento de la demanda de dinero llevaría a un ataque especulativo contra la divisa, que obligaría a abandonar el tipo de cambio fijo y a adoptar un tipo de cambio flexible.

Un aspecto importante de estos modelos es que el ataque contra la divisa siempre ocurriría antes de que el banco central agotara el nivel de sus reservas internacionales en ausencia de especulación, y tendría lugar en una fecha bien definida. Es decir, dada una tasa positiva de crecimiento del crédito interno, los especuladores racionales anticiparían que, en ausencia de especulación, las reservas internacionales se agotarían eventualmente, por lo que preverían el colapso final del sistema y, con el fin de evitar pérdidas, forzarían una crisis antes de que se produjera dicho colapso.

En síntesis, los modelos de primera generación establecen que una creación excesiva de crédito interno por parte del gobierno llevaría a una pérdida gradual de reservas internacionales y, en última instancia, al abandono del tipo de cambio fijo, una vez que el banco central se reconoce incapaz de defender la paridad.

El modelo básico de ataque especulativo está representado por la ecuación:

$$
\frac{M_{t}}{P_{t}}=\alpha_{\theta}-\alpha_{t} i_{t},
$$

donde $\alpha_{\theta}, \alpha_{t} i_{t}>0$.

La ecuación (1) es una función de demanda de dinero, donde la demanda de saldos reales de dinero, $\frac{M_{t}}{P_{t}}\left(M_{t}\right.$ es la oferta monetaria y $P_{t}$ es el nivel de precios), depende inversamente del tipo de interés.

Adicionalmente, la oferta monetaria, cuyo supuesto es que está determinada por la base monetaria, tiene dos componentes: los activos nacionales en poder del banco central o crédito interno, $D_{t}$, y el valor de las reservas internacionales expresado en moneda local, $R_{t}$. Es decir:

$$
M_{t}=D_{t}+R_{t} .
$$

Si el crédito interno crece a una tasa constante, $\mu$, entonces $M_{t}=\mu$, donde $\mu>0$. Adicionalmente, se establece que se respeta la teoría de la paridad del 
poder de compra (PPP), por lo que el nivel de precios locales es igual al nivel de precios en el extranjero, entonces:

$$
P_{t}=P_{t}^{*} E_{t}
$$

donde $P_{t}^{*}$ representa el nivel de precios en el extranjero y $E_{t}$ es el tipo de cambio. La paridad descubierta de la tasa de interés está dada por la ecuación:

$$
i_{t}=i_{t}^{*} \frac{\dot{E}_{t}}{E_{t}}
$$

En esta ecuación $i_{t}^{*}$ representa la tasa de interés en el extranjero y $\dot{E}_{t}$ es la derivada del tipo de cambio.

Al sustituir PPP y la paridad descubierta de la tasa de interés en la demanda de dinero, se obtiene la ecuación del tipo de cambio:

$$
E_{t}=\frac{M_{t}}{\beta}+\frac{\alpha}{\beta} \dot{E}_{t}
$$

En la ecuación (5), $\beta=\alpha_{\theta} P_{t}^{*}-\alpha P_{t}^{*} i_{t}$ y $\alpha=\alpha_{t} P_{t}^{*}$. Con la derivada del tipo de cambio fijo igual a cero, con lo que se obtiene:

$$
\bar{E}_{t}=\frac{M_{t}}{\beta} .
$$

En donde $\bar{E}_{t}$ es el tipo de cambio fijo, por lo que $M_{t}$ es constante y $D_{t}$ aumenta en dirección inversamente proporcional a la reducción de las reservas internacionales, $R_{t}$. Cuando $R_{t}=0$, las autoridades financieras abandonaran el tipo de cambio fijo.

En consecuencia, cualquier incremento del crédito interno debe de financiarse con la emisión de moneda local, lo que genera un proceso inflacionario que provoca la pérdida de valor de dicha moneda. Sin embargo, varios autores insisten en que los agentes económicos no esperan a que las reservas sean mulas, sino que prevén la pérdida de valor de la moneda posterior al agotamiento de las reservas, y adelantan dicho agotamiento mediante un ataque especulativo contra la moneda del país.

Krugman (1979) utiliza el concepto de expectativas inflacionarias para explicar por qué se produce un ataque especulativo antes del agotamiento de las reservas internacionales del banco central. Según este autor, los agentes económicos tienen una expectativa de inflación, que será nula mientras crean en la política cambiaria del gobierno. Es decir que mientras los agentes económicos consideren que la política monetaria va a continuar no se producirá el ataque especulativo.

Ante el ataque especulativo, las autoridades monetarias tienen dos alternativas. Primero, si el banco central tiene todavía reservas, el gobierno puede intervenir en los mercados de divisas para defender la paridad de la moneda local. Segundo, el gobierno puede dejar flotar la moneda local. 


\subsection{Modelos de segunda generación}

Las variables que explicaron las crisis en Latinoamérica a lo largo de la década de los ochenta no se ajustaban a las crisis que se presentaron en el Sistema Monetario Europeo y en México en los noventas, lo que motivo la formulación de nuevos modelos. Estos modelos se conocen como de segunda generación.

Los modelos de segunda generación, como señalan Flood y Marion (1997), se fundamentan en el modelo básico de incoherencia temporal de Kydland y Prescott (1977), que añaden una cláusula de escape dada por la posibilidad de devaluación. En estos modelos, los ataques especulativos pueden aparecer incluso en ausencia de problemas, actuales o futuros, en las variables económicas fundamentales debido a que los ataques se autoconfirman (Self-Fulfilling), es decir, si se espera que ocurra un ataque especulativo terminará ocurriendo, desplazando el tipo de cambio de un equilibrio a otro.

Obstfeld (1994 y 1996), desarrolla un modelo de crisis de balanza de pagos en el que incluye nuevas variables explicativas de las crisis. Este autor señala a las expectativas de devaluación como el detonante del proceso que concluye con la devaluación de la moneda local.

Para ilustrar lo expuesto anteriormente se presenta un modelo basado en Jeanne (1997), en el cual las expëctativas se autoconfirman. La característica de este modelo es que la probabilidad de devaluación depende de las variables económicas fundamentales, pero de una manera no lineal, lo que da lugar a soluciones múltiples.

El modelo parte de la siguiente formulación:

$$
B_{t}=b_{t}-\alpha \pi_{t-1}
$$

donde $b_{t}$ recoge las condiciones objetivas que afectan a $B_{t}$. Además $b_{t}$, está representada por:

$$
b_{t}=\phi_{t-1}+\varepsilon_{t}
$$

En esta ecuación $\phi_{t-1}$ representa las variables económicas fundamentales dadas por $\phi_{t}=E_{t} b_{t-1}$, siendo $E$ el operador de las expectativas de los agentes económicos.

Dado que la racionalidad de las expectativas se cumple en el equilibrio, la probabilidad de devaluación debe ser igual a la probabilidad de que las autoridades monetarias abandonen el tipo de cambio fijo, $\mu$, además de que el beneficio neto de mantenerlo en el periodo siguiente sea negativo, es decir:

$$
\pi_{t}=\mu \operatorname{Prob} L_{t}\left[B_{t+1}<0\right]
$$

Así pues, en este modelo, una crisis cambiaria, definida como una situación en la cual aumenta la probabilidad de devaluación, podría ocurrir si se produce un deterioro de las variables económicas fundamentales, lo que hace que el tipo de cambio fijo sea vulnerable a la especulación que se autoconfirma, ó si se produce un salto en la probabilidad de devaluación.

Calvo y Mendoza (1998) analizan el efecto rebaño (herding) en el comportamiento de los agentes económicos, que crece a medida que se amplía el 
mercado financiero, y que puede provocar crisis en países que no tienen problemas económicos internos.

Es importante resaltar que en los modelos de segunda generación, eỉ origen de las crisis estaría en las expectativas de devaluación de los agentes privados y no en el comportamiento del gobierno. Mientras que en los modelos de primera generación, el origen de la crisis está en la incompatibilidad de la política cambiaría y las políticas monetaria y fiscal del gobierno.

\subsection{Modelos de tercera generación}

Los modelos de tercera generación fueron formalizados por Krugman (1998) y Aghion, Bacchetta y Banarjee (2000, 2001). Estos modelos examinan los efectos que tiene la política monetaria implantada por un gobierno en el estallido de las crisis. Estos modelos no constituyen un campo unificado, más bien ofrecen explicaciones parciales de distintos elementos que caracterizaron las crisis financieras asiáticas.

Un primer grupo de teorías que agrupa a los modelos de tercera generación, enfatiza el problema del riesgo moral (Krugman (1998) y Corsetti, Pesenti y Roubini (1998)). Estos modelos postulan que los gobiernos de los países emergentes avalan, implícitamente, todo tipo de proyectos de inversión, incluidos los altamente riesgosos, lo que da como resultado un exceso de inversión y un creciente endeudamiento con el exterior. Si en un momento determinado se produce una pérdida de confianza por parte de los agentes económicos, debido a cualquier perturbación desfavorable, éstos pensarán que el gobierno no puede satisfacer las garantías implícitas prometidas y se retirarán del país, llevando al país a un colapso de su tipo de cambio y una crisis financiera.

Un segundo grupo de teorías descansan en la fragilidad del sistema financiero de los países (Chang y Velasco (2000 y 2001)). Estos modelos sostienen que la fragilidad en la banca y en el sector financiero reducen el crédito disponible a los agentes económicos, provocando un incremento en la probabilidad de la crisis.

Adicionalmente, los modelos de tercera generación sugieren que la crisis es una combinación de alto endeudamiento, bajas reservas internacionales, incremento en las expectativas de devaluación, tasas de interés que no incentivan la. inversión, o que frenan el endeudamiento, y poca disponibilidad de créditos.

Los modelos de tercera generación dan un lugar especial a la política monetaria ya que, además de tomar la decisión de abandonar o no el tipo de cambio, tienen la facultad de contraer o no el crédito interno. Si los agentes económicos perciben la poca disponibilidad de créditos en moneda local tenderán a endeudarse en moneda extranjera, lo que aumenta su riesgo ante una devaluación.

Adicionalmente, el gobierno para atraer inversión tendera a emitir deuda indexada a una moneda extranjera, lo que reduce el riesgo de los inversionistas ante una devaluación. Esta situación hace que los gobiernos se enfrenten a una situación de liquidez internacional, es decir el gobierii no puede, en el corto plazo, conseguir las divisas necesarias para pagar todas sus obligaciones, lo cual, ante una repentina perdida de confianza, lleva a la liquidación de inversiones, lo que confirma el pesimismo inicial; el resultado será un colapso del tipo de cambio y del sistema financiero. 


\section{Características económicas de México antes de la crisis de 1994}

\subsection{Dos sexenios de crisis}

La reactivación económica en México durante los primeros años de la administración de López Portillo (1976-1982) fue muy rápida. Sin embargo, la deuda externa del sector público pasó de 19,600 millones de dólares a finales de 1976 a 52,961 millones de dólares al finalizar 1982, es decir, se experimentó un incremento del $170 \%$.

El gobierno mexicano creía que mediante la inversión pública aumentaría la inversión privada y el crecimiento económico, y se resolverían los problemas de desempleo y bajos ingresos. Además, las inversiones en la industria petrolera, en poder del Estado, fueron otro elemento que condujo a la contratación de deuda.

A principios de la década de los ochenta, el déficit comercial, la sobrevaluación del tipo de cambio y el déficit fiscal aumentaron de manera continua en México. El déficit financiero del sector público, por su parte, registró incrementos importantes (en 1980 representaba el $7.9 \%$ del PIB). No obstante, de estos elementos, el más decisivo para el estallido de la crisis de 1982 fue el déficit comercial. También, la corrupción y la ineficiencia administrativa agravaron los problemas que se vivían en ese momento. Además, como consecuencia de la política contractiva iniciada por el presidente de Estados Unidos Jimmy Carter y continuada por Ronald Reagan para combatir la inflación, las tasas de interés aumentaron y la liquidez internacional se redujo.

A pesar de esto, a finales de 1980 se decidió mantener la política expansiva para alcanzar tasas de crecimiento elevadas, pero en mayo de 1981 empezó a. disminuir el precio del petróleo, que representaba tres cuartas partes de las exportaciones totales de México en ese momento, lo que provocó la fuga de capitales y una crisis en el país. La pérdida de divisas por la disminución de los precios del petróleo y la fuga de capitales fue compensada con créditos externos costosos, dadas las condiciones de los mercados internacionales, ya que provenían de fuentes de financiamiento de corto plazo.

Entre 1980 y 1982 el déficit fiscal aumentó de un $7.5 \%$ del PIB en 1980, a un $14.1 \%$ en 1981 y a $16.9 \%$ en 1982 . Esto hizo que aumentaran los problemas de las finanzas públicas de México.

La deuda externa mexicana en 1981, de la cual casi el $50 \%$ era de corto plazo representó, junto con la situación económica general, un factor determinante de la fuga de capitales.

Las devaluaciones del peso ocurridas los días 17 y 26 de febrero de 1982, así como los programas de ajuste del 19 de febrero y del 11 de abril, no detuvieron la salida de capitales, aunque produjeron un cambio de signo en la balanza comercial debido a la disminución de la demanda de los bienes importados.

El sexenio del presidente De la Madrid (1982-1988), se inició con un ambiente económico desfavorable: las finanzas públicas eran altamente deficitarias, existía un deterioro en la cuenta corriente, el tipo de cambio estaba subvaluado y se producían constantes renegociaciones de la deuda pública y privada. Todos estos elementos propiciaron un deterioro del nivel de vida de la mayoría de la población y una creciente fuga de capitales. 
Aun cuando la economía mexicana inició, en 1984, un modesto proceso de recuperación, no se podía hacer frente a la deuda externa en las condiciones pactadas. Por ello, se acordó un préstamo sindicado por 3,800 millones de dólares para cubrir el déficit de divisas. Los acreedores internacionales, sin embargo, se oponían a una reestructuración.

Los acontecimientos posteriores -el terremoto de 1985 que destruyó la ciudad de México, la caída de los precios del petróleo en 1986 y la inaccesibilidad a los créditos externos- pusieron al país al borde de la suspensión de pagos, lo que conllevó la renegociación total de la deuda. Las perturbaciones internas y externas que vivió México en 1985 deterioraron, aún más, las expectativas económicas de los inversores. El 7 de septiembre de 1985, finalmente, después de unas muy difíciles negociaciones, se concretó un acuerdo.

A mediados de 1986, se acentuó el deterioro de los indicadores económicos de México. El PIB se contrajo en más del 3\% y México volvía a estar ante la situación de no poder cumplir con su política de deuda externa y ante la necesidad de recursos. De mayo a junio, se produjo un intenso debate sobre la necesidad de una nueva renegociación entre el Gobierno mexicano y los acreedores internacionales, pero sólo la amenaza de una eventual suspensión de pagos convenció a los acreedores para la renegociación.

A través de un acuerdo, entre mayo de 1986 y noviembre de 1987, se canceló deuda por un total de 2,488 millones de dólares, de los cuales 2,116 se tradujeron en nuevas inversiones en diferentes sectores del país. Esto ayudó a reactivar de forma moderada el crecimiento económico de México durante 1987 y 1988, aunque en estos años se experimentó la mayor inflación de la historia del país.

Con el fin de recobrar la confianza de los inversores, el gobierno mexicano puso en marcha en 1987 el Programa de Aliento y Crecimiento (PAC), que pretendía restablecer la confianza de los agentes económicos, corregir las finanzas públicas y disminuir las excesivas transferencias netas de recursos al exterior.

La política adoptada en el manejo del tipo de cambio formó parte de la estrategia gubernamental para absorber las perturbaciones externas, con los habituales efectos inflacionarios. Se produjeron varias devaluaciones: en junio de 1985, un 20\%; en noviembre de 1987, un 40\%; y en enero-febrero de 1988, un $3.2 \%$. La diferencia con las otras devaluaciones (especialmente la de 1987) consistió en que sirvieron para frenar la salida de divisas en lugar de ser una de las consecuencias de ella. De hecho, se intentó mantener el peso subvaluado.

El sexenio del presidente De la Madrid (1982-1988) tuvo que hacer frente al deterioro de las cuentas con el exterior, al déficit público, a la especulación, a la devaluación y a la crisis en general. En realidad, éste fue básicamente un sexenio de crisis. Las políticas implementadas por las autoridades monetarias mexicanas en este sexenio tuvieron como objetivo aliviar las fuertes presiones sobre la balanza de pagos, disminuyendo así la transferencia de recursos al exterior, a costa del crecimiento económico, para cumplir con los acreedores. Esto ocasionó un deterioro progresivo del nivel de vida de la población. 
Cabe destacar el esfuerzo del gobierno hacia el final de este sexenio por reactivar la economía y contener la inflación. La renegociación de la deuda y el Pacto de Solidaridad Económica constituyeron los elementos centrales de la reestructuración de la economía mexicana en la siguiente etapa, el sexenio de Carlos Salinas de Gortari (1988-1994), lo que dio lugar a un período de creciniento con relativa estabilidad hasta finales de 1994.

\subsection{Cambios en las crisis 1988-1994}

A finales del sexenio del presidente De la Madrid y principios del sexenio de Salinas de Gortari (1988-1994), se planteó la reducción de las transferencias netas de recursos al exterior y el hecho de pasar de importador neto de capitales a exportador neto. Dentro de esta política, era esencial reducir el saldo y el pago de la deuda externa como proporción del PIB, especialmente la bancaria. Essta se derivaba sobre todo de la necesidad de afrontar las crisis, financiar el déficit gubernamental y hacer frente a las fugas de capitales, más que con fines de inversión. Para cumplir con este objetivo, el gobierno mexicano llevó a cabo un proceso de desregularización bancaria a finales de 1988 .

El 31 de diciembre de 1988 el saldo de la deuda externa ascendía a 100,384 millones de dólares, de los cuales 81,003.2 millones correspondían al sector público. De éstos, 57,786 millones estaban contratados con la banca comercial internacional. Además, del saldo total de la deuda pública externa, el 99\% se encontraba contratado a largo plazo; el $0.99 \%$ a medio plazo y sólamente el $0.1 \%$ correspondía a deuda de corto plazo (véase la gráfica 1). Esto ofrecía una estructura sin presión de pagos inmediatos. Sin embargo, el aumento de las tasas de interés internacionales, seguían ejerciendo una fuerte presión tanto en las finanzas públicas como en la balanza de pagos, limitando el crecimiento eçonómico de México.

El pago por concepto del servicio de la deuda en 1988 fue de $15,611.7$ millones de dólares, de los cuales 9,258.7 millones correspondían a amortización del principal y $6,353.0$ millones a intereses.

En febrero de 1989 una delegación mexicana viajó a Washington con el fin de plantear a la banca comercial internacional y a los organismos financieros internacionales la reducción del monto de la deuda. Por su parte, las autoridades financieras de los Estados Unidos ya se labían planteado esquemas de reducción de la deuda, mediante el aprovechamiento de los descuentos de la misma en los mercados secundarios. El convenio se firmo en 1989 y consistió en solicitar la reducción del saldo de la deuda, la disminución de la tasa de interés y la concesión de nuevos créditos.

Una vez alcanzado el acuerdo, el 23 de julio de 1989 el presidente Salinas dirigió un discurso al pueblo mexicano en el que anunció que se había solucionado el problema del endeudamiento externo de manera definitiva. El 15 de septiembre de 1989 se dieron a conocer los términos y condiciones de los acuerdos, y el 4 de febrero de 1990 se firmaron en el Palacio Nacional los convenios definitivos.

Adicionalmente, en abril de 1989, el gobierno mexicano eliminó las inversiones obligatorias de todos los recursos captados por la banca comercial mexicana. También se permitió que los bancos determinaran libremente las tasas de interés de sus depósitos. 
Gráfica 1. Deuda externa bruta del sector público.

(saldos al final del período en millones de dólares, sin incluir los Tesobonos)

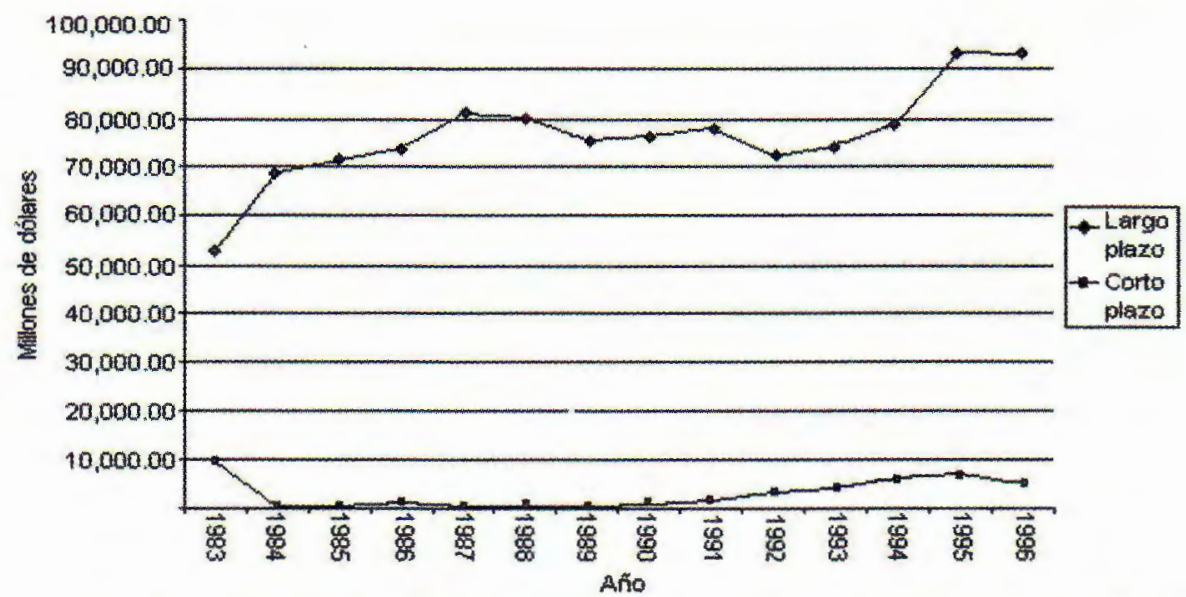

Fuente: Elaboración propia con datos del Sexto Informe de Gobierno de Carlos Salinas de Gortari e informes trimestrales sobre deuda pública de la SHCP.

La deuda externa del sector público experimentó en diciembre de 1989 una disminución real de 4,944 millones de dólares con respecto a diciembre de 1988 . Además, con la firma de los convenios de reestructuración de la deuda se logró que el país regresara a los mercados financieros internacionales.

En resumen, los factores que lograron la reapertura de dichos mercados, tanto para el sector público como para el privado, fueron la contracción del gasto y la inversión pública, la reforma cambiaría, el ajuste patrimonial, el saneamiento de las finanzas públicas, la reducción de las tasas de interés nacionales e internacionales y la reestructuración de la deuda.

La nueva estrategia de endeudamiento externo consistió en no solicitar grandes préstamos y colocar varias emisiones de deuda por montos más modestos. Mediante este proceso, México se convirtió en el principal concentrador de flujos netos de capital hacia América Latina. La inversión extranjera directa pasó de 3,176 millones de dólares en 1989 a 4,389 en 1993, lo que significaba un crecimiento del $38.20 \%$.

Por lo que respecta a la inversión en cartera, esta pasó de 351 millones de dólares en 1989 a 28.919 millones de dólares en 1993, lo que representó un $8.139 \%$ de la inversión extranjera total en ese año, contra un $13.97 \%$ en 1989 , marcando una pronunciada preferencia por este tipo de inversión. Todo ello se tradujo en desequilibrios importantes en la cuenta corriente y de capitales.

El año siguiente, 1990, trajo para México un contexto internacional con demasiadas incertidumbres: la volatilidad de los precios del petróleo, debido al conflicto del Golfo Pérsico, el descenso de la actividad económica en los países industrializados y la depreciación del dólar frente a las divisas de los países desarrollados. A pesar de esos factores y del repunte inflacionario, México destacó por una mayor confianza en el desarrollo de la economía, con lo que la 
inversión extranjera siguió entrando al país. Por todo ello, la evolución de la balanza de pagos reflejó, en conjunto, un desarrollo más favorable que en 1989.

Al finalizar 1990, parecía que el problema del endeudamiento externo había terminado, por lo que los analistas internacionales dejaron de prestar atención a México. Además, durante los primeros años de la administración de Salinas se siguieron poniendo en marcha otros mecanismos, tanto de reducción como de contratación de deuda.

En 1991, el sector público mexicano registró un endeudamiento neto de 2,217.5 millones de dólares. Este endeudamiento provino principalmente de la colocación de bonos, de organismos bilaterales y de la aportación de dinero nuevo de la banca comercial extranjera. Para ese año, el saldo de la deuda pública externa bruta como proporción del PIB representó un $28.39 \%$.

Para 1991 la banca comercial mexicana tenía más del 77\% de los valores gubernamentales, lo que representa un incremento del $332 \%$ respecto a 1985 , mientras que el Banco de México sólo conservaba el 20\%, es decir, un 266\% menos. La demanda creciente de valores gubernamentales por parte de la banca comercial contrastaba con los menores requerimientos financieros del país, debido a la creciente entrada de divisas a México y a los ingresos extraordinarios que se obtuvieron por la venta de las empresas paraestatales.

En junio de 1992, el presidente Salinas anunció la cancelación de 7,171 millones de dólares de bonos a la par y de bonos con descuento, lo que en realidad representaba la anulación contable de deuda por la amortización anticipada, pagada con nuevo endeudamiento.

Entre 1992 y 1993 se generó en México un endeudamiento neto de 2,661.5 millones de dólares, menos los ajustes favorables por variaciones del tipo de cambio igual a 328.7 millones de dólares. Además, se pagaron intereses por un monto de 4,803.9 millones de dólares, lo que significó una transferencia neta de recursos al exterior por 2,104.4 millones de dólares.

Desde 1991 hasta 1994, la economía mexicana continuó registrando un crecimiento moderado. Esto, aunado a la sobrevaluación del tipo de cambio y a la inversión extranjera que recibió México, por la política de anclar el peso al dólar, generó un déficit cada vez mayor en cuenta corriente, principalmente en la balanza comercial.

Además, cabe destacar que en 1987 había comenzado a reducir el nivel de vida de los mexicanos lo que fue decisivo para la incapacidad del mercado mexicano de absorber un crecimiento sostenido de la producción. Es decir, la contracción del mercado interno fue frenando progresivamente el crecimiento del PIB durante la mayor parte del sexenio de Salinas. La pérdida del dinamismo económico antes de 1990, influyó en el ánimo de los inversores, sobre todo en el de los nacionales. Por ello, a partir de 1991 se inició la desconfianza y la fuga de capitales.

En total, en la administración de Salinas entraron al país 101,933 millones de dólares por medio de inversión extranjera. Este solo hecho explica la casi nula necesidad de divisas requeridas en la mayor parte del sexenio, lo que ocasionó la apariencia de que el endeudamiento ya no era un problema. Pero del total de inversión extranjera, un $70.2 \%$ fue en cartera. Así, casi tres cuartas 
partes del déficit de cuenta corriente tuvieron que ser financiadas con inversión especulativa, lo que demuestró la frágil posición de México con respecto al exterior.

La salida de divisas en la cuenta corriente durante el sexenio de Salinas fue de 105,786 millones de dólares. Este nivel de salida de capitales no hubiera sido posible, ni siquiera durante los primeros años, sin una forma de compensación, que fue la cuenta de capital, es decir, dinero ajeno.

A finales de 1993 la situación parecía insostenible para los analistas internacionales. La apreciación del peso había llegado a tal nivel que, dados los indicadores de cuenta corriente, podía generar presiones especulativas devaluatorias. Sin embargo, la aprobación del Tratado de Libre Comercio de América del Norte (NAFTA) creó una visión positiva sobre la economía mexicana.

En 1994, la economía mexicana se enfrentó a una serie de acontecimientos adversos desde principios de año, por lo que el Gobierno centró sus esfuerzos en mantener la estabilidad de los mercados financieros y cambiarios. De hecho, la crisis de diciembre de 1994 en México se había iniciado el 1 de enero de ese mismo año, con el levantamiento armado en Chiapas. A pesar de eso, las reservas internacionales del país continuaron creciendo: habían pasado de 25,334 millones de dólares en diciembre de 1993 a 25,885 millones de dólares en marzo de 1994. Pero el día 23 de marzo de 1994, con el asesinato de Luis Donaldo Colosio, candidato del partido oficial a la presidencia, la situación económica de México comenzó a ser insostenible.

A partir de abril, México aumentó progresivamente las tasas de interés, con la finalidad de mantener niveles de rendimiento atractivos para los inversores. Sin embargo, ante las expectativas de riesgo cambiario, el incremento de las tasas de interés fue insuficiente, por lo que se intentó retener el ahorro en el mercado financiero mediante la emisión de Tesobonos (bonos indexados al dólar), para sustituir los títulos en divisa nacional.

La fuga de capitales durante los primeros dos trimestres de 1994 ascendió a 6,462 millones de dólares y 5,289 millones de dólares respectivamente. Sin embargo, la inversión de cartera siguió afluyendo durante los tres primeros trimestres del año, a pesar del aumento de las tasas de interés en Estados Unidos.

La salida de capitales presionó a la baja las reservas internacionales e incrementó las tasas de interés, lo que representó un grave problema para el gobierno mexicano. Prímero, porque la caída en las reservas ponía en peligro la viabilidad del régimen cambiario y, segundo, porque el incremento en las tasas de interés aumentaba la vulnerabilidad del sistema bancario que tenía un nivel elevado de cartera vencida.

La inversión de cartera pareció reaccionar desfavorablemente ante los acontecimientos del segundo trimestre de 1994, pero en el tercer trimestre. tanto los inversores extranjeros como los nacionales comenzaron a reingresar dólares a la economía mexicana. La fuerte entrada de capitales obligó a las autoridades mexicanas a realizar diversas operaciones de regulación monetaria para atenuar las presiones generadas por dichas entradas. Pero una parte creciente de los recursos obtenidos del exterior se canalizó hacia el sistema bancario. y posteriormente fue colocado en el sector privado con el fin de convertir a la banca de desarrollo en banca de segundo piso. 
El financiamiento por parte de la banca de desarrollo mexicana al sector público llegó a su nivel más bajo en todo el sexenio. Una parte importante de los recursos externos fue intermediada por el sistema bancario mexicano, lo que afectó a sus depósitos y contribuyó a la creciente disponibilidad de recursos para el sector privado, y a la expansión del crédito.

Al inicio del sexenio del presidente Ernesto Zedillo (1994-2000), las autoridades financieras mexicanas parecían tener la convicción de poder evitar el estallido de la crisis. De hecho, dos semanas antes de la devaluación se reiteraba una y otra vez que no habría devaluación. La nueva administración también guardó en secreto el estado de la econonía en ese momento.

El lunes 19 de diciembre de 1994, las autoridades financieras mexicanas decidieron ajustar la paridad cambiaría en un 15\%, con lo que el tipo de cambio pasó de 3.5 a 4.0 pesos por dólar. Esta medida fue tomada como respuesta a la baja de la Bolsa Mexicana de Valores (un 4.15\%), y de que la paridad pesodólar se situaba en el tope máximo de la banda de flotación por segundo día consecutivo.

El incremento de la paridad cambiaría produjo un efecto negativo entre los agentes económicos. Al percibir que el Banco Central mexicano perdía reservas y que la sobrevaluación del peso superaba el $15 \%$, comenzaron a retirar sus capitales del país. Esto provocó una abrupta caída de la bolsa, una pérdida de reservas por valor de 2,500 millones de dólares y una creciente presión especulativa.

A finales de diciembre de 1994, las reservas internacionales en poder del Banco de México se situaron alrededor de los 6,000 millones de dólares, lo que significó que en tan sólo un mes hubo una pérdida de 11,000 millones de dólares. De hecho, llegó un momento en febrero de 1995 en que la posibilidad de agotamiento absoluto de las reservas era cuestión de días. Al mismo tiempo, la fuga de capitales y la salida de inversión de cartera eran mayores que nunca: 1,689 millones de dólares y 7,355 millones, respectivamente, durante el primer trimestre de 1995, a pesar de la devaluación.

En contra de la mayoría del Congreso de su país, el entonces presidente de los Estados Unidos, Bill Clinton, promovió la formación de un paquete financiero en el que participaron el Gobierno de Estados Unidos, el FMI, el Banco Mundial, el Banco Internacional de Pagos y el Banco Interamericano de Desarrollo. Con estos recursos, Ernesto Zedillo puso en marcha el Acuerdo de Unidad para Superar la Emergencia Económica (AUSEE).

Para estabilizar los mercados, el AUSEE contó con recursos financieros por valor de 50,537.4 millones de dólares provenientes de los organismos internacionales. De éstos, 26,797 millones se utilizaron durante 1995. El otorgamiento de la línea de crédito tenía como objetivo convencer a los inversores extranjeros de que existía liquidez suficiente para garantizar las obligaciones financieras de México a corto plazo.

Sin embargo, las nuevas líneas de crédito no lograron reducir la volatilidad de los mercados financieros debido al incremento de las tasas de interés en los países industrializados. Además, la complejidad para obtener los recursos del AUSEE, así como la prolongación del proceso de negociaciones, ocasionó entre los inversores una mayor incertidumbre y una mayor percepción de riesgo con respecto a la economía mexicana. 
Ante estos inconvenientes, el Gobierno mexicano presentó el 9 de marzo de 1995 un nuevo programa encaminado a reforzar el AUSEE. El nuevo plan recibió el nombre de Programa de Acción para Reforzar el Acuerdo de Unidad para Superar la Emergencia Económica (PARAUSEE), y tenía como objetivo principal sacar al país lo más pronto posible de la emergencia económica.

Durante el primer trimestre de 1996, se liquidó el saldo de los Tesobonos. También se anunciaron operaciones de cobertura de divisas de créditos contratados en divisas diferentes del dólar estadounidense.

\section{Ajuste de los modelos a la crisis mexicana}

Habiendo planteado las características económicas y de deuda previas, a la crisis mexicana de 1994, se puede analizar si lo observado para la crisis financiera de México coincide con lo explicado por los modelos teóricos.

Se observa que las variables explicativas de una crisis en los modelos de primera generación no son piezas importantes en la gestación de la crisis mexicana de 1994. Ya que no presentaba déficit públicos preocupantes. Sin embargo, si hubo una disminución en las reservas internacionales en poder del Banco de México antes del colapso del peso, como tiende a ocurrir de acuerdo con estos modelos.

Sobre los modelos de segunda generación, cabe destacar que el incremento en las tasas de interés y en la inflación encarecieron la deuda pública mexicana y mermaron la competitividad del país. Adicionalmente, se observa que los problemas políticos que tuvo México durante 1994 incrementaron las expectativas de devaluación. Así mismo, la estructura de vencimientos de la deuda gubernamental alertó a los agentes económicos sobre la posibilidad de una devaluación.

Por otra parte se observa que México no presentaba problemas de bajo crecimiento o desempleo que pudieran elevar las expectativas de devaluación del mercado, como prevén los modelos de segunda generación.

No obstante, la idea de que hay costos asociados al mantenimiento del tipo de cambio y de que la elevación de estos costos trae consigo la elevación de las expectativas de devaluación, podría aplicarse al caso mexicano. Se observó que México tenía, por ejemplo, problemas comerciales crecientes, por lo que resulta evidente los altos costos de mantener el tipo de cambio.

México tenía algunas deficiencias estructurales, como señalan los modelos de segunda generación, sin embargó, conviene puntualizar el impacto de tales deficiencias ya que algunos países latinoamericanos presentaron problemas similares y no sufrieron ataques especulativos (Argentina) o los sufrieron más tarde, en parte debido al contagio de la crisis asiática como fue el caso de Brasil. Ante este fenómeno se puede afirmar que la economía mexicana no se hubiera enfrentado a la crisis de 1994, si no hubiera habido retroalimentación entre los problemas internos y las expectativas de devaluación.

Con respecto al efecto de rebaño, es difícil determinar el papel de este mecanismo en la generación de la crisis mexicana, por la necesidad de conocer la psicología de los inversionistas. Aunque hay evidencia empírica de que fueron los agentes económicos nacionales los primeros en sacar sus capitales del país, motivados más por rumores que por el conocimiento de la economía mexicana. 
Por lo que respecta a los modelos de tercera generación, antes de la crisis de 1994 se nota un deterioro del sistema financiero mexicano, que queda en evidencia por la restricción en el crédito bancario, por lo que el sector público y privado tuvieron que recurrir a préstamos en el extranjero, con el consabido riesgo cambiario.

Así mismo, el proceso de liberación financiera trajo consigo problemas en el sistema bancario. Las grandes entradas de capital superaron la capacidad operativa del sistema financiero mexicano. Esta situación debilitó la posición financiera de los bancos y, ante la salida de estos capitales, se presentó una crisis bancaria.

Adicionalmente, el uso de fondos externos fue alentado por tasas de interés internas relativamente altas, con las que se procuraba mantener baja la inflación en un contexto de rápido crecimiento. Pero la disponibilidad y el acceso a capital externo, el marco financiero más liberal y permisivo, y la escasa supervisión por parte de las autoridades financieras mexicanas facilitaron la inversión y el endeudamiento excesivos de las empresas, configurándose así una situación de vulnerabilidad financiera nacional.

\section{Conclusiones}

Los modelos de primera generación no son aplicables a la crisis financiera que sufrió México en 1994. Sin embargo, los modelos de segunda generación podrían explicar dicha crisis en el sentido de que estudian cómo el deterioro de los indicadores económicos fundamentales pudieron derivar en la crisis financiera, aunque no fueron determinantes en el estallido de dicha crisis.

Lo ocurrido en México en 1994 muestra que, si hay una total libertad para el movimiento de capitales externos, las autoridades monetarias no pueden prevenir el sobreendeudamiento del sector publico y privado, lo que deteriora la calidad de las carteras de los bancos locales; además, la cuantiosa afluencia de capital tiende a producir una excesiva expansión del crédito. Si, simultáneamente surgen desequilibrios en la cuenta corriente, debido al elevado gasto financiado por capitales externos, el deterioro del balance externo y el debilitamiento del sector financiero son dos caras del mismo proceso. Lo anterior, llevó al establecimiento de controles prudenciales y al mejoramiento de la calidad del capital que ingresaba a México como medidas para evitar las crisis.

Otro factor a considerar en la crisis mexicana, relacionado con los problemas en los bancos y el sistema financiero, es que éstos han permanecido después de que las cconomías se han recuperado, ya que el establecimiento del marco institucional necesario para un desarrollo sano del sector financiero ha tomado varios años y no se ha podido consolidar.

\section{Bibliografía}

Aghion, P., P. Bacchetta, and A. Banerjee (2001). Currency crises and monetary policy in an economy with credit constraints.European Economic Review, 45(7), pp. 1121-1150.

Aghion, P., P. Bacchetta, and A. Banerjee (2000). A simple model of monetary policy and currency crises. European Economic Review, 44 (4-6), pp. 728-738.

Bustelo, P. (1998a). El FMI y la crisis en Asia oriental, ¿quiebra de un modelo de desarrollo? VI Jornadas de Economía Crítica, Málaga. 
Bustelo, P. (1998b). The East Asian Financial Crises: An Analytical Survey. Documento de trabajo, ICE-UCM.

Calvo, G. (1996). Varieties of Capital-Market Crises. University of Maryland.

Calvo, G. A. and E. G. Mendoza (1998). Rational Behavior and the Globalization of Securities Markets. University of Maryland.

Chang, R. and A. Velasco (2001). Monetary policy in a dollarized economy where balance sheets matter. Journal of Development Economics, 66(2), pp. 445-464.

Chang, R. and A. Velasco (2000). Banks, debt maturity and financial crises. Joumal of International Economics, 51 (1), pp. 169-194.

Connolly, M. B. and D. Taylor (1984). The Exact Timing of the Collapse of an Exchange Rate Regime and its Impact on the Relative Price of Traded Goods. Journal of Money. Credit and Banking, 16(1), pp. 194-207.

Corsetti, G., P. Pesenti, and N. Roubini (1998). What Caused the Asian Currency and Financial Crisis? New York University.

Edwards, S. (1989). Temporary Terms-of-Trade Disturbances, the Real Exchange Rate and the Current Account. Economica. New Series, 56(223), pp. 343-357.

Esquivel, G. and F. Larrain (1998). Explaining Currency Crises. Harvard Institute for International Development.

Fischer, S. (1998). The Asian Crisis: A View from the IMF. Fondo Monetario Internacional.

Flood, R. and M. Garber (1984). Collapsing Exchange Rate Regimes: Some Linear Examples. Journal of International Economics, 1, pp. 1-13.

Flood, R., M. Garber, and C. Kramer (1996). Collapsing Exchange Rate Regimes: Another Linear Example. Journal of International Economics, 41(3/4), pp. 223-234.

Flood, R. and N. Marion (1997). The size and timing of devaluations in capital-controlled economies. Journal of Development Economics, 54(1), pp. 123-147

Frankel, J. A. (1998). The Asian Model, the Miracle, the Crisis and the Fund. New York University.

Green, R. (1988). La deuda externa de México, 1973-1987: De la abundancia a la escasez de créditos. México, D. F. Secretaria de Relaciones Exteriores: Nueva Imagen.

Green, R. (1976). El endeudamiento público externo de México 1940-1973. México, D. F., El Colegio de México.

Griffith, J. (1996). La crisis del peso mexicano. Revista de la CEPAL, pp. 151-170.

Guidotti, P. and M. Kumar (1991). Domestic Public Debt of Externally Indebted Countries. Fondo Monetario Internacional, Documento de trabajo.

Guillén, A. H. (1990), El sexenio del crecimiento cero, México 1982-1988. México, D. F. ERA.

Guillén, A. H. (1996a). Los mercados financieros y el derrumbe del neoliberalismo mexicano. Revista de Investigación Económica, pp. 271-298.

Guillén, A. H. (1996b). Opciones frente a la crisis de México.Comercio Exterior, 46, pp. 321-328.

Goldstein, M. (1998). The Asian Financial Crisis. Institute for International Economics.

Instituto Nacional de Estadística, Geografía e Informática. Banco de Información Económica, Base de datos.

Jeanne, O. (1997). Are currency crises self-fulfilling?: A test. Joumal of International Economics, 43(3-4), pp. 263-286.

Krugman, P. (1979). A Model of Balance-of-Payments Crises. Journal of Money, Credit and Banking, 11(13), pp. 311-325.

Krugman, P. (1998a). What Happened to Asia? Massachusetts Institute of Technology.

Krugman, P. (1998b). Currency Crises. Massachusetts Institute of Technology.

Kydland, F. and E.Prescott (1977). Rules Rather than Discretion: The Inconsistency of Optimal Plans. The Journal of Political Economy, 85(3), pp. 473-492.

Obstfeld, M. (1994). The Logic of Currency Crises. Caffiers Economíques et Monótaires, 43, pp. 189-212.

Obstfeld, M. (1996). Models of Currency Crises with Self-Fulfilling Features.European Economic Review, 40, pp. 1037-1047. 
Ortiz, G. (1995). La reforma financiera y la desincorporación bancaria. Una visión de la modernización de México. México, D. F., Fondo de Cultura Económica.

Ortiz. G. and J. Serra Puche (1986). La Carga de la deuda externa de México. Estudios Económicos, pp. 20-29.

Presidencia de la República (1994). Sexto informe de gobierno de Carlos Salinas de Gortari. México, D. F.

Presidencia de la República (1995). Primer informe de gobierno de Ernesto Zedillo Ponce de León. México, D. F,

Radelet, S. and J. Sachs (1998). The Onset of the East Asian Financial Crisis. Harvard Institute for International Development.

Salant, S. and D. Henderson (1978). Market Anticipation of Government Policy and the Price of Gold.Journal of Political Economy, 86, pp. 627-648.

Sous, L. (1996). Crisis Económico-Financiera 1994-1995. México, D. F., Fondo de Cultura Económica.

Werner, A. (1994). Expectativas de realineación y la banda cambiaria óptima. El Trimestre Económico, 1, pp. 409-432. 